\title{
Surgical management of polycystic ovarian disease
}

\author{
Deepal S Weerasekera ${ }^{1}$ \\ Sri Lanka Journal of Obstetrics and Gynaecology 2009; 31: 53-54
}

Key words: polycystic ovarian disease, surgical management

\section{Introduction}

Polycystic ovarian disease (PCOD) is the most common endocrine disorder in women. It's prevalence among subfertile women is around $20 \%$. The etiology of PCOD remains unclear. It has been suggested that PCOD is an X-linked dominant condition ${ }^{1}$. Clinical features of PCOD include oligomenorrhoea, hirsutism, acne, obesity, and subfertility. Biochemically serum androgens, lutinising hormone and LH/FSH ratio are elevated. Ultrasonographic features of polycystic ovaries include presence of 12 or more follicles in each ovary measuring 2-9 $\mathrm{mm}$ in diameter and/or increased ovarian volume $(>10 \mathrm{ml})$.

\section{History of surgical treatment}

Stein and Leventhal first reported wedge resection of the ovaries in PCOD associated with subfertility. They observed restoration of regular menstruation in approximately $80 \%$ and spontaneous conception in about $50 \%$ of the patients so treated. However this procedure was associated with a high percentage of ovarian and periadnexal adhesions that may even lead to infertility in some patients. Also this procedure is associated with a substantial loss of ovarian tissue which may lead to premature ovarian failure. Therefore this procedure has been abandoned in today's practice after the initial experience for a limited time.

Introduction of laparoscopic ovarian drilling (LOD) reawakened the interest in surgical treatment of PCOD patients unresponsive to comiphene citrate. Ovarian drilling is the creation of multiple holes through the ovarian capsule using a pointed monopolar or bipolar diathermy electrode or with laser surgery.

\section{Mechanism of action of LOD}

Mechanism of action of LOD is unclear. The beneficial effect is apparently due to destruction of

\footnotetext{
${ }^{1}$ Clinical Director, "Prarthana" Centre for ART, Rajagiriya.

E-mail: deepalsenaka@yahoo.co.uk
}

the androgen producing stroma. Following LOD there is a decrease in circulating androgen and free testosterone concentrations. The serum LH rises immediately after the procedure and then decreases. The effect on FSH is variable and less pronounced. These endocrine changes occur rapidly and are sustained for several years resulting in recruitment of new follicles and restoration of ovulation in most of the subjects ${ }^{2}$.

\section{The technique}

LOD is considered for patients with polycystic ovaries resistant to treatment with clomiphene citrate. Ovarian drilling can be performed by laparotomy, laparoscopy or transvaginal hydrolaparoscopy. It is recommended to make four drill holes in each ovary using $40 \mathrm{~W}$ diathermy applying for 4 seconds for each drill. The electrode should be inserted perpendicular to the ovary to a depth of about $4-5 \mathrm{~mm}$. It is important to hold the ovary away from bowel and tube to avoid thermal damage to these organs. Once the procedure is performed the ovary can be cooled by instilling saline. Ovarian drilling when performed by laparoscopy can be done as an out-patient procedure.

\section{Ovulation and conception after ovarian drilling}

Considerable variation exists in the reported outcomes of LOD in patients with PCOD resistant to clomiphene citrate. In a review of ten studies in which cumulative ovulation and pregnancy rates at 12 months after LOD showed $82.7 \%$ and $64.8 \%{ }^{3}$. There are additional advantages provided by LOD. Patients who were resistant to clomiphene citrate may respond to this medication after the procedure. Therefore in patients who do not ovulate spontaneously following LOD, treatment should be initiated with clomiphine citrate. In a review of 223 LOD performed by author $22 \%$ conceived spontaneously within the first 3 months following surgery. In women who did not conceive ovulation induction was done with clomiphene citrate and intrauterine insemination was performed. Further $16 \%$ conceived with 3 treatment cycles.

Also in women undergoing IVF, LOD improves the outcome. In these patients there is more orderly 
growth of follicles, serum estradiol concentrations are lower, the rate of cycle cancellation is less, and the incidence of ovarian hyperstimulation syndrome (OHSS) is reduced ${ }^{4}$.

\section{Comparison with exogenous gonadotrophins}

Alternative management strategy to LOD in clomiphene resistant PCOD is the treatment with gonadotrophins. Extensive monitoring is necessary with this treatment due to high sensitivity of polycystic ovaries to exogenous gonadotrophins leading to OHSS. The majority of the studies found no difference in the rates of ovulation, pregnancy and miscarriages between the two modalities of treatment. The multiple pregnancy rates are lower with ovarian drilling than with gonadotrophins. The beneficial improvement in menstrual regularity, reproductive performance, and endocrine effects of LOD may continue for years. Subsequent medical induction of ovulation becomes easier following ovarian surgery.

\section{Prediction of response to LOD}

Although high ovulation and pregnancy rates following LOD in patients with clomiphene citrate resistant PCOD have been widely documented, $20-30 \%$ of such cases may fail to respond to surgical therapy. Presence or absence of acne, the menstrual pattern, and ovarian volume did not influence the outcome of LOD. A BMI greater than $35 \mathrm{~kg} / \mathrm{m}^{2}$, serum testosterone concentration greater than $4.5 \mathrm{nmol} / 1$ and free androgen index more than 15 are associated with a poor response. The LH/FSH ratio was found to be the strongest predictor of ovarian response after electrocautery $^{5}$. Therefore it is important to advice the obese patients to loose weight by diet and exercise before undergoing surgery.

\section{Complications}

Complications can occur due to anesthesia, establishment of surgical access i.e. laparoscopy, laparotomy or transvaginal hydrolaparoscopy, and the drilling procedure itself. Bleeding can occur from the drilling sites, lacerations can occur in the mesoovarian ligament during grasping to stabilize the ovary for drilling. Using excessive amounts of energy can destroy a large number of follicles leading to decreased ovarian reserve. The use of excessive amounts of energy with an electrode inserted too deeply into the ovary may cause damage to hilar vessels resulting in pre-mature ovarian failure. Post operative adhesions can be a significant complication especially when laparotomy is performed for surgical access.

\section{Conclusions}

Ovarian drilling is a treatment option in clomiphene citrate resistant PCOD patients. When performed via laparoscopy this is a relatively simple procedure which can be done on an out-patient basis. This procedure offers several advantages when compared with the alternative mode of treatment which is gonadotrophins administration. Following advantages of LOD are well documented:

- Over $70 \%$ of patients ovulate spontaneously following the procedure

- More than $50 \%$ conceive without the need of intensive cycle monitoring as necessary when gonadotrophins are used.

- Procedure provides long term effect

- Eliminates the risk of multiple pregnancy

- Eliminates the risk of OHSS associated with gonadotrophins

- It creates a favorable response to stimulation with clomiphene citrate or gonadotrophins if their use becomes necessary

Despite these advantages ovarian drilling is a surgical procedure done under anesthesia and can be associated with complications specially when performed on obese patients. Until more effective and safer new treatment options become available ovarian drilling is strongly considered in the current management of $\mathrm{cc}$ resistant PCOD.

\section{References}

1. Speroffl FM. Anovulation and polycystic ovary. In: Clinical gynecologic endocrinology and infertility. 7th edn. Lippincott Williams \& Wilkins, Baltimore, MA, 2005 pp 470-83.

2. Amer SA, Gopalan V, Li TC Ledger WL, Cook ID. Long term follow-up of patients with polycystic ovarian syndrome after LOD. Clinical outcome. Hum Reprod 2002; 17: 2035-42.

3. Saleh AM, Khalil HS. Review of nonsurgical and surgical treatment and the role of insulin sensitizing agents in the management of infertile women with polycystic ovary syndrome. Acta Obstet Gynecol Scand 2004; 83: 614-21.

4. Tozer AJ, Al-Shawaf T, Zosmer A, et al. Does laparoscopic ovarian diathermy affect the outcome of IVF-embryo transfer in women with polycystic ovarian syndrome? A retrospective comparative study. Hum Reprod 2001; 16: 91-5.

5. Amer SA, Li TC, Cooke ID. A prospective dose finding study of the amount of thermal energy required for laparoscopic ovarian diathermy. Hum Reprod 2003; 18: 1693-8. 